\title{
A feasibility pilot study of the effects of neurostimulation on dysphagia recovery in Parkinson's Disease [version 1; peer
} review: 1 approved with reservations, 1 not approved]

\author{
Ayodele Sasegbon (D1), Ulrike Hammerbeck (D1), Emilia Michou (Di),2, Ivy Cheng (iD1, \\ Mengqing Zhang ${ }^{1}$, Charlotte James ${ }^{1}$, Shaheen Hamdy (iD)1 \\ ${ }^{1}$ Division of Diabetes, Endocrinology and Gastroenterology, University of Manchester, Manchester, Greater Manchester, Stott Lane, \\ Salford M6 8HD, UK \\ 2Department of Speech and Language Therapy, University of Patras, Patras, Greece
}

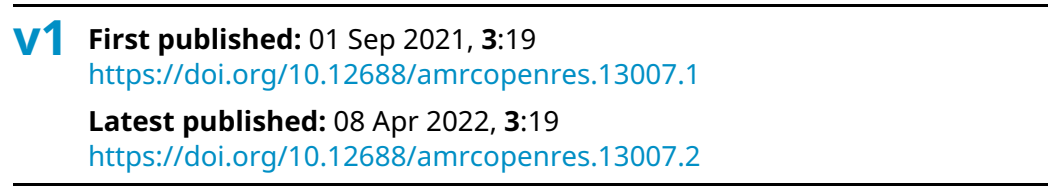

\section{Abstract}

Introduction: Dysphagia often occurs during Parkinson's disease (PD) and can have severe consequences. Recently, neuromodulatory techniques have been used to treat neurogenic dysphagia. Here we aimed to compare the neurophysiological and swallowing effects of three different types of neurostimulation, $5 \mathrm{Hertz}(\mathrm{Hz})$ repetitive transcranial magnetic stimulation (rTMS), $1 \mathrm{~Hz} \mathrm{rTMS}$ and pharyngeal electrical stimulation (PES).

Method: 12 PD patients with dysphagia were randomised to receive either $5 \mathrm{~Hz}$ rTMS, $1 \mathrm{~Hz}$ rTMS, or PES. In a cross-over design, patients were assigned to one intervention and received both real and sham stimulation. Patients received a baseline videofluoroscopic (VFS) assessment of their swallowing, enabling penetration aspiration scores (PAs) to be calculated for: thin fluids, paste, solids and cup drinking. Swallowing timing measurements were also performed on thin fluid swallows only. They then had baseline recordings of motor evoked potentials (MEPs) from both pharyngeal and (as a control) abductor pollicis brevis (APB) cortical areas using single-pulse TMS. Subsequently, the intervention was administered and post interventional TMS recordings were taken at 0 and 30 minutes followed by a repeat VFS within 60 minutes of intervention. Results: All interventions were well tolerated. Due to lower than expected recruitment, statistical analysis of the data was not undertaken. However, with respect to PAs swallowing timings and MEP amplitudes, there was visual separation in a positive direction between active and sham groups for all interventions.

Conclusion: PES, $5 \mathrm{~Hz}$ rTMS and $1 \mathrm{~Hz}$ rTMS are tolerable interventions in PD related dysphagia. Due to small patient numbers no definitive conclusions could be drawn from the data with respect to individual interventions improving swallowing function and comparative
Open Peer Review

\begin{tabular}{|c|c|c|c|c|}
\hline & 1 & 2 & 3 & 4 \\
\hline $\begin{array}{l}\text { version } 2 \\
\text { (revision) } \\
08 \text { Apr } 2022\end{array}$ & & & $\begin{array}{c}\checkmark \\
\text { view }\end{array}$ & $\begin{array}{c}? \\
\text { view }\end{array}$ \\
\hline $\begin{array}{l}\text { version } 1 \\
01 \text { Sep } 2021\end{array}$ & $\underset{\text { view }}{\boldsymbol{X}}$ & $?$ & & \\
\hline
\end{tabular}

1. Jin Woo Park ID, Dongguk University Ilsan Hospital, Gyeonggi-do, South Korea

2. Corinne A. Jones, Dell Medical School, The University of Texas at Austin, Austin, USA The University of Texas at Austin, Austin, USA

3. Paul Muhle ID, University Hospital Muenster, Muenster, Germany

4. Massimiliano Todisco ID, IRCCS Mondino

Foundation, University of Pavia, Pavia, Italy

Any reports and responses or comments on the article can be found at the end of the article. 
effectiveness between interventions. Larger future studies are needed to further explore the efficacy of these neuromodulatory treatments in Parkinson's Disease associated dysphagia.

\section{Keywords}

Dysphagia, Swallowing, rTMS, PES, Parkinson's

PARKINSON'S FINDACATE.

JOIN US.
This article is included in the Parkinson's UK

gateway.

Corresponding author: Ayodele Sasegbon (ayodele.sasegbon@manchester.ac.uk)

Author roles: Sasegbon A: Data Curation, Formal Analysis, Investigation, Project Administration, Visualization, Writing - Original Draft Preparation, Writing - Review \& Editing; Hammerbeck U: Data Curation, Investigation, Project Administration, Writing - Review \& Editing; Michou E: Conceptualization, Funding Acquisition, Investigation, Project Administration, Writing - Review \& Editing; Cheng I: Data Curation, Investigation, Validation, Writing - Review \& Editing; Zhang M: Data Curation, Investigation, Writing - Review \& Editing; James C: Investigation; Hamdy S: Conceptualization, Funding Acquisition, Supervision, Writing - Review \& Editing

Competing interests: Shaheen Hamdy is chief scientific officer, a shareholder, and a board member of Phagenesis Ltd., a company that focuses on dysphagia therapies, specifically pharyngeal electrical stimulation. Shaheen Hamdy has also received research funding from MRC, Wellcome Trust, Stroke Association, and NIHR to explore brain stimulation strategies to treat post-stroke dysphagia. All other authors declare no conflicts of interest.

Grant information: Funding for this study was provided by Parkinson's UK (grant reference G-1401).

Copyright: @ 2021 Sasegbon A et al. This is an open access article distributed under the terms of the Creative Commons Attribution License, which permits unrestricted use, distribution, and reproduction in any medium, provided the original work is properly cited.

How to cite this article: Sasegbon A, Hammerbeck $U$, Michou E et al. A feasibility pilot study of the effects of neurostimulation on dysphagia recovery in Parkinson's Disease [version 1; peer review: 1 approved with reservations, 1 not approved] AMRC Open Research 2021, 3:19 https://doi.org/10.12688/amrcopenres.13007.1

First published: 01 Sep 2021, 3:19 https://doi.org/10.12688/amrcopenres.13007.1 


\section{Introduction}

Parkinson's disease (PD) is a common neurodegenerative condition of unclear aetiology wherein there is a build-up of Lewy Bodies within dopaminergic regions of the brain ${ }^{1}$. These Lewy Bodies are primarily composed of the protein alpha synuclein and cause damage to the internal workings of neurones ${ }^{2,3}$. As the disease progresses, there is an increasing burden of pathological protein deposition and an associated decline in neuronal function ${ }^{1,4,5}$. From the point at which a diagnosis of PD is made, patients tend to exhibit an increasing number of symptoms in a predictable manner. As a result, symptomatic scales such as the Hoehn and Yahr scale ${ }^{6}$ are often used to classify PD severity. Epidemiological studies have shown $\mathrm{PD}$ is present in up to $4 \%$ of people over 55 years of age $\mathrm{e}^{7,8}$. Although the limb and gait disturbances caused by PD are common and well $\mathrm{known}^{5}$, PD is also recognised to cause dysphagia ${ }^{9}$. Dysphagia commonly occurs in patients with $\mathrm{PD}^{10}$, with up to $82 \%$ of patients developing dysphagia at some point along their illness journey ${ }^{11}$. PD can cause dysphagia directly or indirectly. The direct pathway occurs as a result of Lewy body related damage to swallowing centres within the brain $^{12}$. Conversely, the indirect pathway is due to damage to non-motor brain areas which results in dementia ${ }^{13}$ which causes dysphagia $^{14}$.

At present the management of dysphagia in PD is geared towards compensating for neurological damage with interventions such as dietary modification, altering the consistency of fluids and the use of dopaminergic medications ${ }^{15,16}$. However, a body of evidence exists in support of invasive deep brain stimulation (DBS) for the treatment of PD motor symptoms ${ }^{17}$. DBS delivered to the subthalamic nucleus or globus pallidus is effective at ameliorating motor dysfunction up to 12 months after treatment ${ }^{17}$. There is little data on whether DBS can treat PD dysphagia. Beyond this, neuromodulatory interventions constitute new and emerging developments in the treatment of neurogenic dysphagia. Novel and increasing applied techniques include pharyngeal electrical stimulation (PES) and repetitive transcranial magnetic stimulation (rTMS). PES is a technique whereby a catheter containing two electrodes is inserted transnasally or per-orally into the pharynx. The application of an electric current results in stimulation of sensory afferents supplying the pharynx and increased sensory inflow into brain areas including the sensory and motor cortices ${ }^{18}$. RTMS, by contrast, is a centrally acting as opposed to a peripherally acting technique. It uses a strong electromagnet to pulse magnetic energy at targeted parts of the brain including the swallowing motor cortical areas ${ }^{19}$. High-frequency rTMS (5 Hertz or greater) causes increases in pharyngeal motor cortical neurological excitability ${ }^{20}$ while low frequency (1 Hertz) rTMS causes a suppressive effect ${ }^{21}$.

Very few non-invasive neurostimulatory studies have been performed in PD with even fewer being performed in the field of dysphagia. Regarding PES, no study has been performed investigating the effects of PES on PD related dysphagia. However, PES has been used in numerous studies as a treatment for post-stroke dysphagia (PSD) ${ }^{22,23}$. A meta-analysis of these studies shows PES is able to improve swallowing performance $^{24}$. Moreover, a single randomised controlled trial utilising high-frequency rTMS in PD dysphagia was performed in 2019 by Khedr et al. ${ }^{25}$. In that study, rTMS was shown to lead to improvements in a functional dysphagia scale (the Arabic dysphagia handicap score) and pharyngeal transit time for thin fluids and solids ${ }^{25}$. Despite the dearth of rTMS swallowing studies in PD, numerous rTMS studies have been performed in the field of PD limb motor function. While their findings are not directly translatable, they do give an idea of potential swallowing therapeutic effects. These studies have employed both low $(1 \mathrm{~Hz})$ and high frequency $(5 \mathrm{~Hz})$ cortical targeted rTMS. High frequency (excitatory rTMS) is thought to be able to excite areas of the motor cortex which are thought to be suppressed secondary to PD mediated damage ${ }^{26}$. By contrast, it is thought that $1 \mathrm{~Hz}$ (suppressive) rTMS blocks maladaptive neuronal activity in the motor cortex thereby allowing beneficial neuroplastic changes to occur ${ }^{26}$. A meta-analysis of the motor effects of rTMS has shown low-frequency rTMS is able to improve PD limb symptoms ${ }^{27}$. High-frequency rTMS trended towards but did not achieve significance ${ }^{26}$.

In light of the lack of studies in the field of swallowing in $\mathrm{PD}$, we aimed to compare the neurophysiological and videofluoroscopic (VFS) swallowing behavioural effects of three neurostimulatory techniques, low-frequency rTMS $(1 \mathrm{~Hz})$, high-frequency rTMS $(5 \mathrm{~Hz})$ and PES in patients with dysphagia secondary to PD. Our objective was to generate data establishing proof of concept, feasibility, safety and tolerability.

\section{Methods}

The study was designed as a triple intervention, two-armed crossover, randomised controlled feasibility trial (Figure 1). Although the initial aim was to recruit 66 participants, the COVID-19 pandemic made this unfeasible. For each of the three interventions; $1 \mathrm{~Hz}$ rTMS, $5 \mathrm{~Hz}$ rTMS and PES, active stimulation was compared with sham. Over the course of the study, each patient was randomly allocated to one of the three interventions and attended the neuro-motility laboratory on two occasions separated by at least one week. During their initial attendance they received either real or sham stimulation and during their second attendance, the alternative.

The study was assessed and granted ethical approval by the Yorkshire \& The Humber - Leeds East Research Ethics Committee (17/YH/0031) and registered on ClinicalTrials.gov (NCT03253354).

\section{Patient recruitment}

Participants were recruited from general neurology clinics, dedicated PD clinics in Salford Royal Hospital (Salford, UK) and PD UK branch meetings.

Inclusion criteria required that patients be diagnosed with PD at least two years prior to the start of the study. Furthermore, patients needed to complain of symptoms of dysphagia, be able to give informed consent and have moderate to severe PD (Hoehn and Yahr Scale II to IV) ${ }^{6}$. 


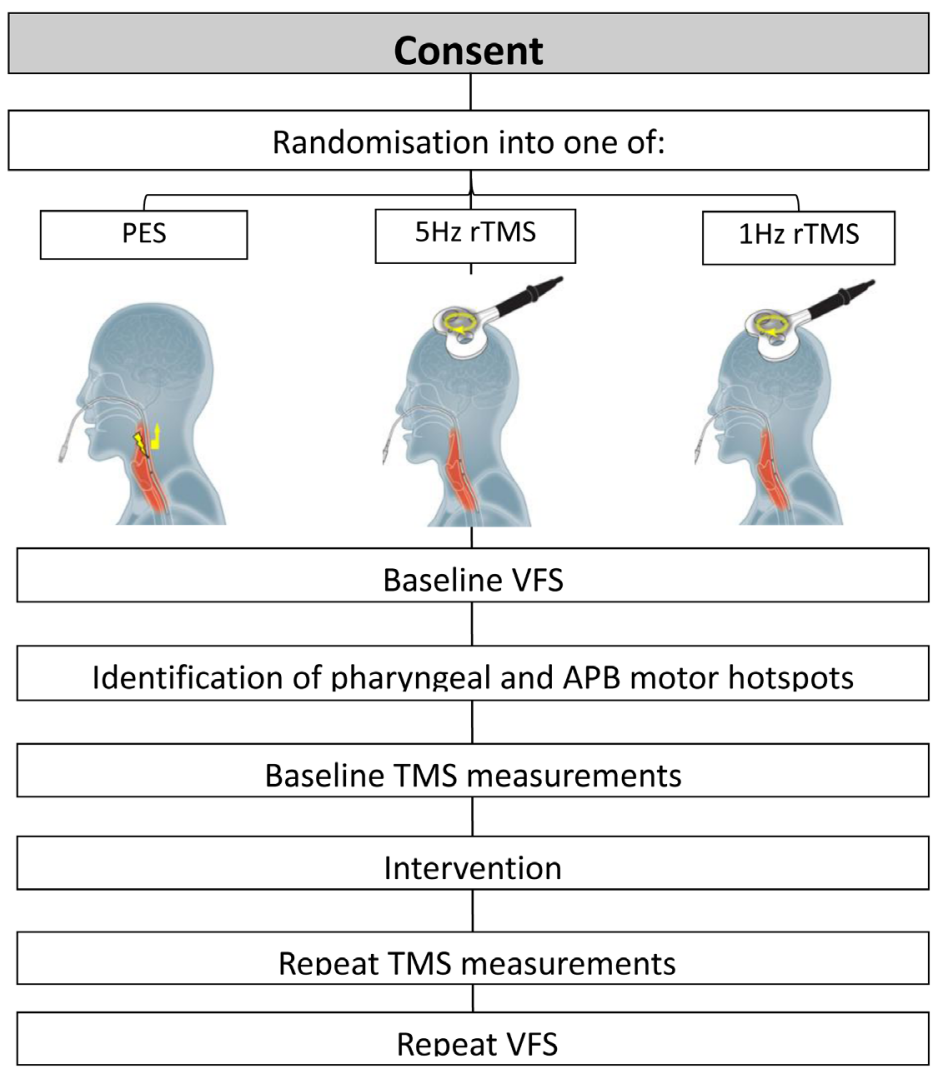

Figure 1. Flowchart illustrating study protocol.

The study exclusion criteria were designed to remove patients: with non-PD causes of dysphagia, with PD mimicking pathologies (multi-system atrophy etc.), lacking capacity to give informed consent and possessing contra-indications for TMS (epilepsy, cardiac pacemakers and metal within the head or neck).

After consenting participants, randomisation to intervention and treatment arms (active or sham) was performed using the statistical website Randola (http://www.rando.la/). Participants then received a screening VFS but only progressed into the study if they had a PAS of 2 or more, indicating swallowing dysfunction. Patients were blinded (so far as possible) to the intervention they received.

\section{Symptomatology and activities of daily living}

Following randomisation, researchers spoke to participants and completed a Hoehn and Yahr scale ${ }^{6}$ and Schwab and England activities of daily living (ADL) scale ${ }^{28}$.

\section{Outcome measures}

The primary outcome measure for the study was any change between pre- and post-interventional VFS assessed PAS for barium of a 'thin fluid' consistency. PAS constitutes the current gold standard for dysphagia assessment in clinical practice and in research ${ }^{29}$. Cumulative PAS scores were calculated, for primary and secondary PAS outcome measures (see below) and for each thickness or task of barium sulphate swallowed.

Secondary outcome measures included:

1. Change in PAS scores with paste consistency, solid consistency (biscuit covered with barium sulphate) and cup drinking of thin barium sulphate fluid.

2. Swallowing timing measurements during thin fluid swallowing, including oral transit time (OTT), pharyngeal transit time (PTT) and pharyngeal response time (PRT).

3. Change in pharyngeal motor evoked potential (PMEP) amplitudes (see study procedures below). Changes in PMEP amplitudes have been shown by previous studies in the field to be correlated with changes in neuronal excitability within the swallowing motor cortex $^{30}$.

\section{Study Procedures}

Electromyography. Electromyography (EMG) recordings (allowing measurements of motor evoked potentials) were obtained from the pharynx and the abductor pollicis brevis (APB). Pharyngeal recordings were made using a trans-nasally inserted intraluminal catheter (Gaeltec, Isle of Skye, UK) as described before $^{31}$. APB EMG signals for recording APB MEPs were used as a control and acquired as previously reported ${ }^{32}$. 
Videofluoroscopy (VFS). VFS recordings were obtained with the assistance of trained radiographers. Participants were seated following which the X-ray source and detector were positioned such that lateral views of oropharyngeal structures could be obtained. Images were recorded at 30 frames per second.

Participants were then asked to swallow 10 thin liquid boluses with a volume of $5 \mathrm{ml}$ (barium sulphate w/v ratio of $60 \%$, equivalent to a IDDSI value of 0). Subsequently, they were asked to swallow 3 boluses of a paste consistency (w/v ratio of $40 \%$ achieved with 'Resource Thicken Up Clear' (Nestle, UK), the equivalent of IDDSI 3) and 3 solid swallows (IDDSI 7). Finally, participants were asked to drink two $50 \mathrm{ml}$ aliquots of thin liquid (IDDSI 0). Barium sulphate (E-Z-Paque, UK) was mixed with water or spread over the surface of solids so as to enable VFS visualisation of boluses. Participants' VFS PAS data were analysed by a speech therapist blinded to the group assignment. PAS values were obtained for every primary and secondary clearing swallow performed. A primary swallow was defined as the first swallow performed when a bolus was ingested, while secondary or clearing swallows were the subsequent swallow that participants performed to clear any residue. Swallowing timing measurements for thin fluid swallows (OTT, PTT and PTR) were also performed by the same blinded speech and language therapist (IC).

During each study session, participants had baseline and post interventional VFS recordings. As a safety feature of the study, VFS was stopped if a participant was noted to aspirate more than $50 \%$ of bolus volume on 3 consecutive swallows ( 3 consecutive PAS scores of 8).

Single-pulse transcranial magnetic stimulation. Single-pulse TMS was used to elicit motor evoked potentials (MEPs) from pharyngeal and APB motor cortical hotspots. Pulses were delivered using a figure-of-eight electromagnetic coil $7 \mathrm{~cm}$ in diameter, with a field strength of 2.2 Tesla, connected to a Magstim Bistim Unit (Magstim, Whitland, UK).

When in use, the coil was held flat against a disposable surgical cap placed over a participant's head at an angle of 45 degrees. Motor mapping was performed as has been described in several published studies $^{31,33}$. Single-pulse TMS was also used to measure PMEP and APB MEP amplitudes. This was done by delivering 10 pulses of stimulation at $120 \%$ of the resting motor threshold (RMT) of the pharyngeal or APB motor areas being studied.

Pharyngeal electrical stimulation. PES was delivered using a $3.2 \mathrm{~mm}$ intraluminal catheter (Gaeltec, Isle of Skye, UK) positioned within the pharynx. The catheter was connected to a signal generator (Digitimer model DS7, Hertfordshire, UK) and a trigger generator (Digitimer Neurology system, Hertfordshire, $\mathrm{UK})^{34}$.

Electrical stimulation was delivered at an intensity determined by the patient's initial sensory threshold and maximum tolerated sensory threshold. The initial sensory threshold was defined as the intensity of electrical stimulation at which a participant first feels they are being stimulated. The maximum tolerated sensory threshold was defined as the electrical intensity at which patients experienced discomfort. To establish these two thresholds the signal generator current was increased in increments of approximately $0.1 \mathrm{~mA}$ each second until patients stated that they could feel a sensation in their throat. The intensity at which this occurred was noted and the process repeated twice more. The mean of the three values was then calculated. The maximum tolerated intensity was determined by increasing the electrical intensity further until patients stated that it felt uncomfortable. The intensity of pharyngeal stimulation was set at $75 \%$ of the difference between the two values ${ }^{34}$.

Active PES was delivered at a frequency of $5 \mathrm{~Hz}$ for 10 minutes $^{34}$. For sham PES, the intraluminal catheter was inserted but no electrical stimulation was delivered.

Repetitive transcranial magnetic stimulation. RTMS was performed using a Magstim super rapid generator (Magstim, Whitland, UK) connected to a $7 \mathrm{~cm}$ figure-of-eight coil. Highfrequency excitatory rTMS was performed by positioning the coil over the pharyngeal motor cortical area with the lowest RMT (the 'dominant' swallowing hemisphere) and delivering 250 pulses at $5 \mathrm{~Hz}$ at an intensity of $90 \%$ of $\mathrm{RMT}^{23}$. Low-frequency suppressive rTMS was also delivered over the pharyngeal motor cortical area, again with the lowest RMT, 600 pulses at a frequency of $1 \mathrm{~Hz}$ and an intensity of $120 \% \mathrm{RMT}^{35}$. Sham rTMS was delivered using the coil tilt technique ${ }^{36}$ where the subject could feel the coil on their scalp and noise of the stimuli, but no energy was delivered to the brain beneath.

Protocol. During each session, patients were first taken to the VFS suite for measurements of their PAS swallowing baseline as described above. Subsequently, they were escorted to the neurophysiology laboratory and seated in a chair. A disposable surgical cap was placed over their heads and secured with medical tape. The location of their cranial vertex was then identified and marked as has been described in previous studies $^{36}$. APB electrodes and an intraluminal pharyngeal catheter were then positioned. Following this, single-pulse TMS was used to locate pharyngeal motor cortical hotspots bilaterally and the APB motor cortical hotspot on the hemisphere with the lowest pharyngeal RMT. RMTs over pharyngeal and APB areas were determined as has been described in previous studies ${ }^{32}$.

Baseline PMEP and APB MEP measurements were obtained by delivering 10 pulses of single-pulse TMS over pharyngeal motor areas bilaterally and the APB area over the 'dominant' pharyngeal hemisphere. Following this, either real or sham: $1 \mathrm{~Hz}$ rTMS, $5 \mathrm{~Hz}$ rTMS or PES was administered. Repeat MEP measurements were then obtained immediately after the intervention and 30 minutes after the intervention. Lastly, participants were taken to the VFS suite for a repeat set of swallowing measurements. A flow chart of the key points of the study protocol can be seen in Figure 1. 


\section{Data analysis}

As this study was intended to be a small pilot study exploring the feasibility of using neurostimulatory techniques to induce beneficial changes in swallowing function, only descriptive statistics including means, medians and standard deviations (SD), were used to compare each active treatment (5 Hz rTMS, PES and $1 \mathrm{~Hz}$ rTMS) to sham. Hoehn and Yahr and Schwab and England ADL scores were also compared between groups.

Penetration aspiration scores. The PAS with the highest numerical value was recorded for each swallow before being added together for each category (thin fluid, paste, solid and cup drinking) to give cumulative penetration aspiration scores ${ }^{23}$. Cumulative scores were converted into percentage differences from individual baseline.

Swallowing timing measurments. OTT, PTT and PRT values were obtained from VFS recordings for all interventions for thin fluid swallows before being converted into percentage changes from baseline.

Motor evoked potentials. MEP amplitudes were measured in microvolts $(\mu \mathrm{V})$. MEP latencies were measured as the time in milliseconds (ms) from the point at which a TMS pulse was delivered to the onset of a MEP. MEP amplitude and latency analysis were performed on a desktop computer (DELL, Berkshire UK) using the program Signal (Version 4.0; Cambridge Electronic Design Ltd, Cambridge, UK). The mean of each set of 10 PMEP and APB MEP amplitudes and latencies (at baseline, 0 mins and 30 mins), were obtained before being converted to percentage changes from baseline.

All statistical calculations were performed on a personal computer (DELL, Berkshire, UK) using SPSS statistics (Version
23, IBM, NY, USA). A P value of 0.05 or less was considered to represent statistical significance.

\section{Results}

Patient recruitment commenced in 2019 and was stopped in 2020 during the COVID-19 pandemic due to the mandated cessation of research particularly, as in this case, research that has the potential to be aerosol-generating.

Twelve people with PD (pwPD) were consented and took part in the study (10 males and 2 females with a mean age of $70( \pm 8)$ years $)$ Table 1. Five patients were randomly allocated to the $5 \mathrm{~Hz}$ rTMS group (4 male 1 female), 3 to the PES group ( 3 males) and 4 to the $1 \mathrm{~Hz}$ rTMS group (3 males 1 female). Mean ages in the $1 \mathrm{~Hz}$ rTMS, $5 \mathrm{~Hz}$ rTMS and Pes groups were $71( \pm 8), 67( \pm 3)$ and $75( \pm 12)$. No adverse events occurred or were reported from participants during and after the studies.

The mean Hoehn and Yahr scores in the $1 \mathrm{~Hz}, 5 \mathrm{~Hz}$ rTMS and PES rTMS groups were $2.9( \pm 0.3), 2.1( \pm 0.6)$ and $1.8( \pm 0.3)$, respectively. The mean Schwab and England ADL score in the $1 \mathrm{~Hz}, 5 \mathrm{~Hz}$ rTMS and PES groups were 68\% $( \pm 13), 80 \%$ $( \pm 12)$ and $87 \%( \pm 6)$.

\section{Cortical parameters}

Seven participants had a dominant right-hemispheric pharyngeal motor area and 5 had a dominant left hemisphere. These remained stable across studies.

The mean pharyngeal RMT over the dominant hemisphere was $77 \%( \pm 9 \%)$ and $69 \%( \pm 11 \%)$ over the non-dominant hemisphere. The mean APB RMT was $43 \%( \pm 13 \%)$.

Using the cranial vertex as a reference point from which to calculate $\mathrm{x}$ and $\mathrm{y}$ co-ordinates, mean cortical pharyngeal

Table 1. Demographic data for study participants.

\begin{tabular}{|c|c|c|c|c|c|c|}
\hline Participants & Intervention & Sex & Age & Time since PD diagnosis & Hohen and Yarr & Schwab and England \\
\hline 1 & $1 \mathrm{~Hz}$ & $\mathrm{M}$ & 78 & 4 & 2.5 & 0.7 \\
\hline 2 & $5 \mathrm{~Hz}$ & $\mathrm{M}$ & 63 & 3 & 2 & 0.8 \\
\hline 3 & $5 \mathrm{~Hz}$ & $\mathrm{~F}$ & 70 & 3 & 1.5 & 0.8 \\
\hline 4 & $\mathrm{PES}$ & $\mathrm{M}$ & 62 & 5 & 1.5 & 0.8 \\
\hline 5 & $1 \mathrm{~Hz}$ & $\mathrm{M}$ & 60 & 8 & 3 & 0.8 \\
\hline 6 & $1 \mathrm{~Hz}$ & $\mathrm{M}$ & 70 & 5 & 3 & 0.7 \\
\hline 7 & $1 \mathrm{~Hz}$ & $\mathrm{~F}$ & 76 & 6 & 3 & 0.9 \\
\hline 8 & $5 \mathrm{~Hz}$ & $\mathrm{M}$ & 66 & 2 & 2 & 0.9 \\
\hline 9 & $\mathrm{PES}$ & $\mathrm{M}$ & 83 & 3 & 2 & 0.9 \\
\hline 10 & $5 \mathrm{~Hz}$ & $\mathrm{M}$ & 67 & 7 & 2 & 0.9 \\
\hline 11 & $\mathrm{PES}$ & $\mathrm{M}$ & 81 & 6 & 2 & 0.9 \\
\hline
\end{tabular}


motor areas were located at $\mathrm{x}=3.9 \mathrm{~cm}( \pm 1.1 \mathrm{~cm})$ and $\mathrm{y}=2.6 \mathrm{~cm}( \pm 1.7 \mathrm{~cm})$ over the right hemisphere and $\mathrm{x}=-2.5 \mathrm{~cm}( \pm 3.6 \mathrm{~cm})$ and $\mathrm{y}=2.5 \mathrm{~cm}( \pm 1.7 \mathrm{~cm})$ over the left hemisphere. APB motor areas were located at $x=4.7( \pm 0.5 \mathrm{~cm})$ and $\mathrm{y}=1.3( \pm 0.9 \mathrm{~cm})$ over the right hemisphere and $\mathrm{x}=-4.8 \mathrm{~cm}$ $( \pm 1.4 \mathrm{~cm})$ and $\mathrm{y}=1.9 \mathrm{~cm}( \pm 2.1 \mathrm{~cm})$ over the left hemisphere.

\section{Penetration aspiration scores}

Mean and median cPAs for each group can be seen in Table 2 .

Thin fluids. Mean percentage differences from baseline PAs for thin fluids in the $1 \mathrm{~Hz}$ rTMS, $5 \mathrm{~Hz}$ rTMS and PES groups were 2.09 (SD: 35.00), 0.49 (SD: 24.50) and -10.53 (SD: 18.23 ) in the active arms compared with 53.57 (SD: 87.77),
18.97 (SD: 57.83) and 103.25 (SD: 171.36) respectively in the sham arms (Figure 2).

Paste. In the active arms mean percentage differences from baseline for the $1 \mathrm{~Hz}$ and PES groups were -16.67 (SD: 23.57) and -19.05 (SD: 32.99) compared with -5 (SD: 0) and 55.56 (SD: 69.39) in the sham arms (Figure $3 \mathrm{~A}+\mathrm{B}$ ). The $5 \mathrm{~Hz}$ rTMS group could not be analysed as all swallows with paste consistency were $<2$ (hence normal) for both active and sham arms for all time points.

Solid. In the PES group, the mean percentage diference from baseline was -20.0 (SD: 34.64) in the active arm and 122.22 (SD: 107.15) in the sham arm (Figure 3 C). Solid swallows in the

\begin{tabular}{|c|c|c|c|c|c|c|}
\hline & & & \multicolumn{2}{|c|}{ Mean } & \multicolumn{2}{|c|}{ Median } \\
\hline & & & Pre & Post & Pre & Post \\
\hline \multirow{8}{*}{$1 \mathrm{HZ}$} & \multirow{4}{*}{ Active } & Thin & $18.8 \pm 5.9$ & $18.0 \pm 2.1$ & 21.5 & 15.0 \\
\hline & & Paste & $4.5 \pm 1.7$ & $3.5 \pm 0.0$ & 4.5 & 3.5 \\
\hline & & Solid & $3.0 \pm 0.0$ & $3.0 \pm 0.0$ & 3.0 & 3.0 \\
\hline & & Cup drinking & $5.0 \pm 3.5$ & $3.0 \pm 0.0$ & 4.0 & 3.0 \\
\hline & \multirow{4}{*}{ Sham } & Thin & $14.0 \pm 8.4$ & $16.0 \pm 1.1$ & 15.5 & 14.5 \\
\hline & & Paste & $3.8 \pm 1.0$ & $3.5 \pm 0.4$ & 3.5 & 3.0 \\
\hline & & Solid & $3.3 \pm 0.5$ & $3.3 \pm 0.2$ & 3.0 & 3.0 \\
\hline & & Cup drinking & $6.0 \pm 3.7$ & $3.0 \pm 0.0$ & 5.5 & 3.0 \\
\hline \multirow{8}{*}{$5 \mathrm{HZ}$} & \multirow{4}{*}{ Active } & Thin & $12.3 \pm 3.3$ & $13.0 \pm 5.6$ & 11.0 & 11.0 \\
\hline & & Paste & $3.0 \pm 0.0$ & $3.0 \pm 0.0$ & 3.0 & 3.0 \\
\hline & & Solid & $3.0 \pm 0.0$ & $3.0 \pm 0.0$ & 3.0 & 3.0 \\
\hline & & Cup drinking & $6.8 \pm 3.0$ & $3.6 \pm 1.3$ & 7.0 & 3.0 \\
\hline & \multirow{4}{*}{ Sham } & Thin & $12.8 \pm 4.7$ & $13.6 \pm 6.1$ & 11.0 & 10.0 \\
\hline & & Paste & $3.0 \pm 0.0$ & $3.0 \pm 0.0$ & 3.0 & 3.0 \\
\hline & & Solid & $3.0 \pm 0.0$ & $3.0 \pm 0.0$ & 3.0 & 3.0 \\
\hline & & Cup drinking & $4.6 \pm 1.9$ & $4.3 \pm 2.2$ & 4.0 & 4.0 \\
\hline \multirow{8}{*}{ PES } & \multirow{4}{*}{ Active } & Thin & $12.3 \pm 5.9$ & $10.3 \pm 2.5$ & 10.0 & 10.0 \\
\hline & & Paste & $6.7 \pm 6.4$ & $4.0 \pm 1.7$ & 3.0 & 3.0 \\
\hline & & Solid & $5.3 \pm 4.0$ & $3.3 \pm 0.6$ & 3.0 & 3.0 \\
\hline & & Cup drinking & $4.0 \pm 0.0$ & $3.5 \pm 0.7$ & 4.0 & 3.5 \\
\hline & \multirow{4}{*}{ Sham } & Thin & $10.0 \pm 7.0$ & $12.3 \pm 4.0$ & 13.0 & 13.0 \\
\hline & & Paste & $3.0 \pm 0.0$ & $4.7 \pm 2.1$ & 3.0 & 4.0 \\
\hline & & Solid & $3.0 \pm 0.0$ & $6.7 \pm 3.2$ & 3.0 & 8.0 \\
\hline & & Cup drinking & $4.5 \pm 2.1$ & $5.0 \pm 4.2$ & 4.5 & 5.0 \\
\hline
\end{tabular}



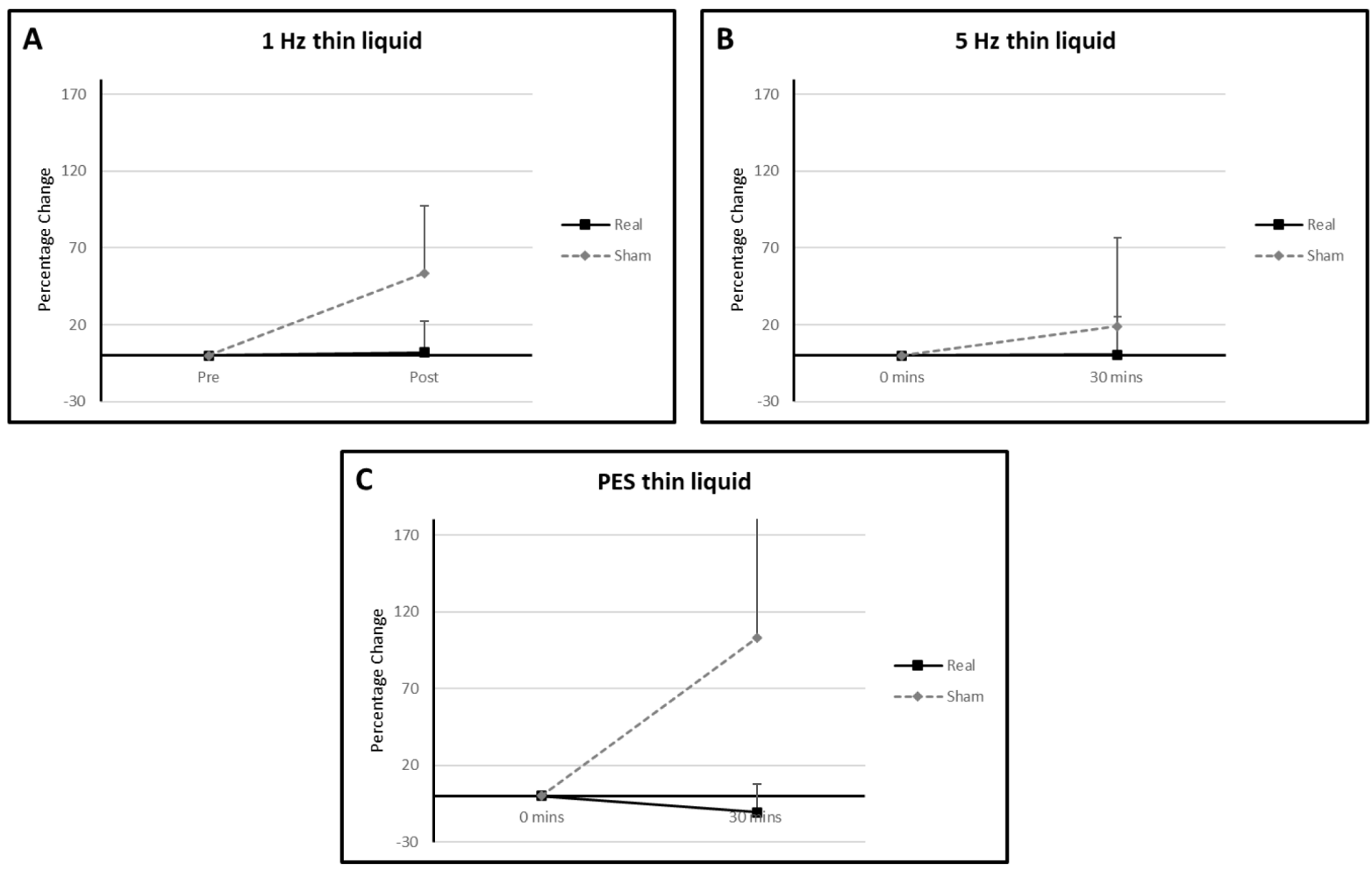

Figure 2. Graphs of percentage differences in PAs for thin fluid in the (A) $1 \mathrm{~Hz}$ rTMS, (B) $5 \mathrm{~Hz}$ rTMS and (C) PES interventional groups. Error bars illustrate standard deviations at each data point.

$5 \mathrm{~Hz}$ and $1 \mathrm{~Hz}$ rTMS groups resulted in PAs values of $<2$ (hence normal) in both active and sham arms. As such no analysis could be performed.

Cup drinking (IDDSI 0). With regards to cup drinking, mean percentage difference in means from baseline in the active 1 $\mathrm{Hz}$ rTMS, $5 \mathrm{~Hz}$ rTMS and PES groups were 0 (SD: 0), -32.29 (SD: 28.94) and -12.5 (SD: 17.68) respectively compared to -24.44 (SD: 21.43), -4.17 (SD: 54.65) and 0 (SD: 47.14) respectively in the sham arms (Figure $3 \mathrm{D}+\mathrm{E}$ ).

\section{Swallow timing results}

Raw timing data for thin fluids can be seen in Table 3 .

Oral transit time. Mean percentage changes in OTT from baseline in the active $1 \mathrm{~Hz}$ rTMS, $5 \mathrm{~Hz}$ rTMS and PES groups were 16.0 (SD: 42.46), 3.38 (SD: 16.75) and 0.01 (SD: 57.02) and -20.26 (SD: 28.62), 9.02 (SD: 26.70) and 3.71 (SD: 64.56) respectively.

Pharyngeal response time. Percentage changes from baseline in the $1 \mathrm{~Hz}$ rTMS, $5 \mathrm{~Hz}$ rTMS and PES groups were 24.78 (SD: 40.80), 9.29 (SD: 22.26) and -2.83 (SD: 19.58) in the active arms respectively. In the sham arms values were 38.0 (SD: 59.91), 17.44 (SD: 21.84) and 21.86 (SD: 28.46) respectively.
Pharyngeal transit time. Mean PTT percentage changes from baseline in the acive arms of the $1 \mathrm{~Hz}$ rTMS, $5 \mathrm{~Hz}$ rTMS and PES groups were 11.83 (SD: 8.53), 0.66 (SD: 29.79) and 36.72 (SD: 83.61) respectively and 4.53 (SD: 16.52), 24.25 (SD: $35.63)$ and 30.64 (SD: 34.10) in the sham arms respectively (Figure 4 B).

\section{Motor evoked potentials}

Mean values for baseline MEP amplitudes and latencies can be seen in Table 4. Comparing percentage changes in amplitudes between 'dominant' and 'non-dominant' pharyngeal motor hemispheres did not reveal a significant difference for $1 \mathrm{~Hz}$ rTMS, $5 \mathrm{~Hz}$ rTMS or PES (Paired T-Test: $\mathrm{T}_{5}=0.99, \mathrm{P}=0.37$, $\mathrm{T}_{9}=0.75, \mathrm{P}=0.47$ and $\left.\mathrm{T}_{5}=1.76, \mathrm{P}=0.14\right)$. Hence data were merged to produce a combined hemispheric value as previously reported $^{23,35}$.

\section{Amplitudes \\ Pharyngeal}

Mean percentage change from baseline PMEP amplitudes in the active arm of the $1 \mathrm{~Hz}$ rTMS group were -2.01 (SD: 34.58) at 0 minutes and 31.55 (SD: 85.11) at 30 minutes compared to sham values of 17.30 (SD: 31.55) and 24.34 (SD: 40.70) (Figure $5 \mathrm{~A}+\mathrm{C}$ ). In the $5 \mathrm{~Hz}$ rTMS group values in the active arm were 14.98 (SD: 28.43) at 0 minutes and 3.52 (SD: 37.95) at 30 minutes compared to -3.83 (SD: 26.99) and -16.09 (SD: 36.36) in the sham arm. In the active arm of the PES 

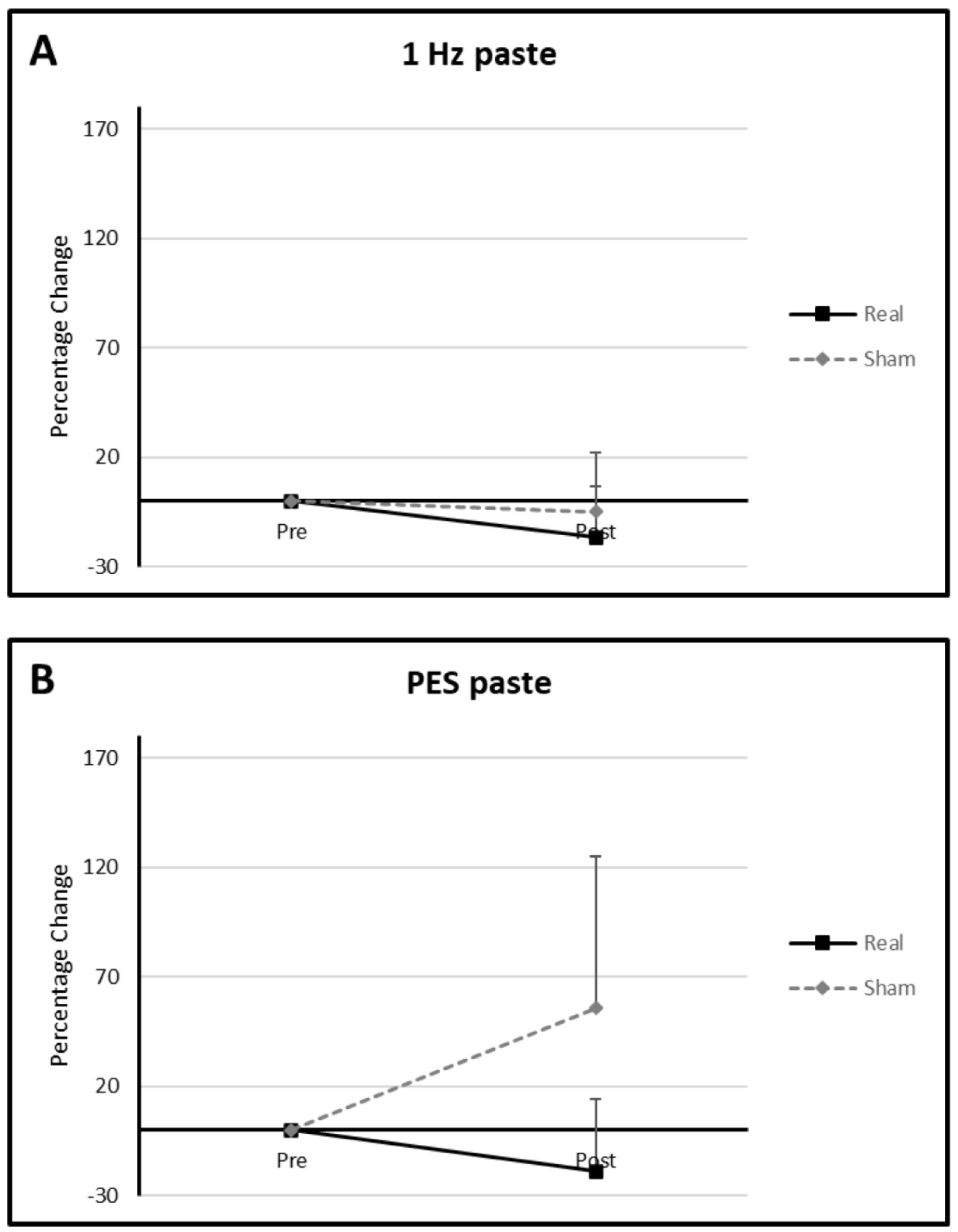

Figure 3. Graphs of percentage differences in PAs for paste consistency in the in the (A) $1 \mathrm{~Hz}$ rTMS and (B) PES interventional groups.

group, values at 0 and 30 minutes were 9.73 (SD: 36.58) and 15.01 (SD: 35.34) compared to 3.93 (SD: 31.92) and -6.63 (SD: 41.17) in the sham arm.

$\underline{\text { APB }}$

Mean percentage changes from baseline for APB MEP amplitudes in the active arm of the $1 \mathrm{~Hz}$ rTMS group were -26.49
(SD: 61.25$)$ at 0 minutes and -43.77 (SD: 53.02 ) at 30 minutes compared to sham values of 35.58 (SD: 33.97) and 32.30 (SD: 35.78) (Figure $5 \mathrm{~B}+\mathrm{D}$ ). In the active arm of the $5 \mathrm{~Hz}$ rTMS interventional group percentage changes from baseline were -13.56 (SD: 60.01) at 0 minutes and 25.68 (SD: 57.79) at 30 minutes contrasted with 3.16 (SD: 69.86) and 18.32 (SD: 83.34) in the sham arm. In the PES group, values at 0 and 30 
Table 3. Swallowing timing data.

\begin{tabular}{|l|r|r|r|}
$\mid$ \\
\end{tabular}

minutes in the active arm were -35.98 (SD: 50.94) at 0 minutes and -49.73 (SD: 68.54 ) at 30 minutes compared to -13.90 (SD: 61.10) and 71.55 (SD: 82.23).

\section{Latencies}

Pharyngeal

Mean percentage change from baseline PMEP latencies in the active arm of the $1 \mathrm{~Hz}$ rTMS group at 0 and 30 minutes were -6.99 (SD: 9.89) and -2.20 (SD: 6.38) compared to sham values of 5.88 (SD: 5.17) and -3.73 (SD: 4.80) (Figure $5 \mathrm{~A}+\mathrm{C}$ ). In the $5 \mathrm{~Hz}$ rTMS group, values in the active arm at 0 and 30 minutes were 3.35 (SD: 9.23) and 4.53 (SD: 9.35) compared to -2.73 (SD: 9.13) and 1.07 (SD: 8.60) in the sham arm. In the active arm of the PES group, values at 0 and 30 minutes were
1.49 (SD: 4.05) and -3.41 (SD: 4.88) compared to -0.42 (SD: 4.69) and -2.57 (SD: 1.07) in the sham arm.

$\underline{\mathrm{APB}}$

Mean percentage changes from baseline for APB MEP latencies in the active arm of the $1 \mathrm{~Hz}$ rTMS group were -1.63 (SD: 5.62 ) at 0 minutes and 0.82 (SD: 5.64) at 30 minutes compared to sham values of -1.61 (SD: 2.45) and -5.54 (SD: 5.00) (Figure $5 \mathrm{~B}+\mathrm{D}$ ). In the active arm of the $5 \mathrm{~Hz}$ rTMS interventional group percentage changes from baseline were 1.97 (SD: 9.44) at 0 minutes and -2.48 (SD: 4.45) at 30 minutes compared to 5.31 (SD: 7.98) and 1.05 (SD: 4.51) in the sham arm. In the PES group, values at 0 and 30 minutes in the active arm were -1.99 (SD: 7.60 ) at 0 minutes and 1.87 (SD: 4.18) at 30 minutes compared to -8.75 (SD: 11.02) and 9.09 (SD: 8.90).

\section{Discussion}

All neuro-stimulatory procedures were well tolerated, implying clinical feasibility with no adverse effects being reported by study participants.

\section{PAs}

Interestingly, across all interventions there was a clear graphical separation between active and sham results, with active stimulation consistently having a lower PAs, and hence appearing to be more physiological beneficial, than sham. It may be that, were more patient data available, statistical analysis and clarity on efficacy may have been possible for one or more interventions. Our findings share some similarities with the results of the only rTMS study performed in PD related dysphagia. In 2019 Khedr et al studied 33 patients with PD and found the application of $20 \mathrm{~Hz}$ rTMS to the hand motor cortex led to improvements in pharyngeal transit time for thin fluids and solids ${ }^{25}$. However, no significant differences were seen regarding PAS values.

More broadly, a meta-analysis conducted in 2015 by Chou et al demonstrated that high-frequency rTMS led to improvements in PD related limb motor dysfunction ${ }^{37}$. However, it should be recognised that the picture regarding the use of high-frequency rTMS to treat PD motor symptoms is a relatively mixed one with another meta-analysis by Shukla et al not showing a clear benefit ${ }^{26}$. In the literature, while there are no studies applying $1 \mathrm{~Hz}$ rTMS to PD dysphagia, a metaanalysis of the effects of $1 \mathrm{~Hz}$ rTMS on motor symptoms in PD showed a significant post interventional improvement ${ }^{26}$. However, similar to the mixed picture for high-frequency rTMS, a recent meta-analysis did not show that $1 \mathrm{~Hz}$ rTMS can induce motor improvement ${ }^{37}$, therefore, no firm conclusions can be made.

\section{Swallowing timing}

The visual improvements in PRT observed for thin fluids particularly in the $1 \mathrm{~Hz}$ rTMS and PES groups are comparable to the improvement in PTT for solids observed by Khedr et al. in $2019^{25}$. These results, imply that both excitatory (PES in this study and $20 \mathrm{~Hz}$ rTMS in the Khedr study) and inhibitory $(1 \mathrm{~Hz}$ rTMS) neurostimulation have the potential to affect swallowing physiology and by so doing improve swallowing function. 
Table 4. Mean baseline cortical pharyngeal and cortical APB MEP amplitudes in microvolts $(\mu \mathrm{V})$ and latencies in milliseconds (ms).

\begin{tabular}{|c|c|c|c|}
\hline & 5 Hz rTMS & PES & $1 \mathrm{~Hz}$ rTMS \\
\hline \multicolumn{4}{|c|}{ MEP amplitudes $(\mu V)$} \\
\hline Cortical pharyngeal & \multirow{2}{*}{$\begin{array}{c}97.5 \pm 72.4 \\
1300.3 \pm 1500.1\end{array}$} & \multirow{2}{*}{$\begin{array}{c}63.4 \pm 37.4 \\
321.5 \pm 179.5\end{array}$} & \multirow{2}{*}{$\begin{array}{c}160.0 \pm 114.3 \\
1323.9 \pm 2118.5\end{array}$} \\
\hline Cortical APB & & & \\
\hline \multicolumn{4}{|c|}{ MEP latencies (ms) } \\
\hline Cortical pharyngeal & \multirow{2}{*}{$\begin{array}{c}8.6 \pm 0.7 \\
25.1 \pm 2.7\end{array}$} & \multirow{2}{*}{$\begin{array}{l}8.4 \pm 0.7 \\
21.5 \pm 2.0\end{array}$} & \multirow{2}{*}{$\begin{array}{c}9.3 \pm 1.1 \\
23.9 \pm 1.1\end{array}$} \\
\hline Cortical APB & & & \\
\hline
\end{tabular}
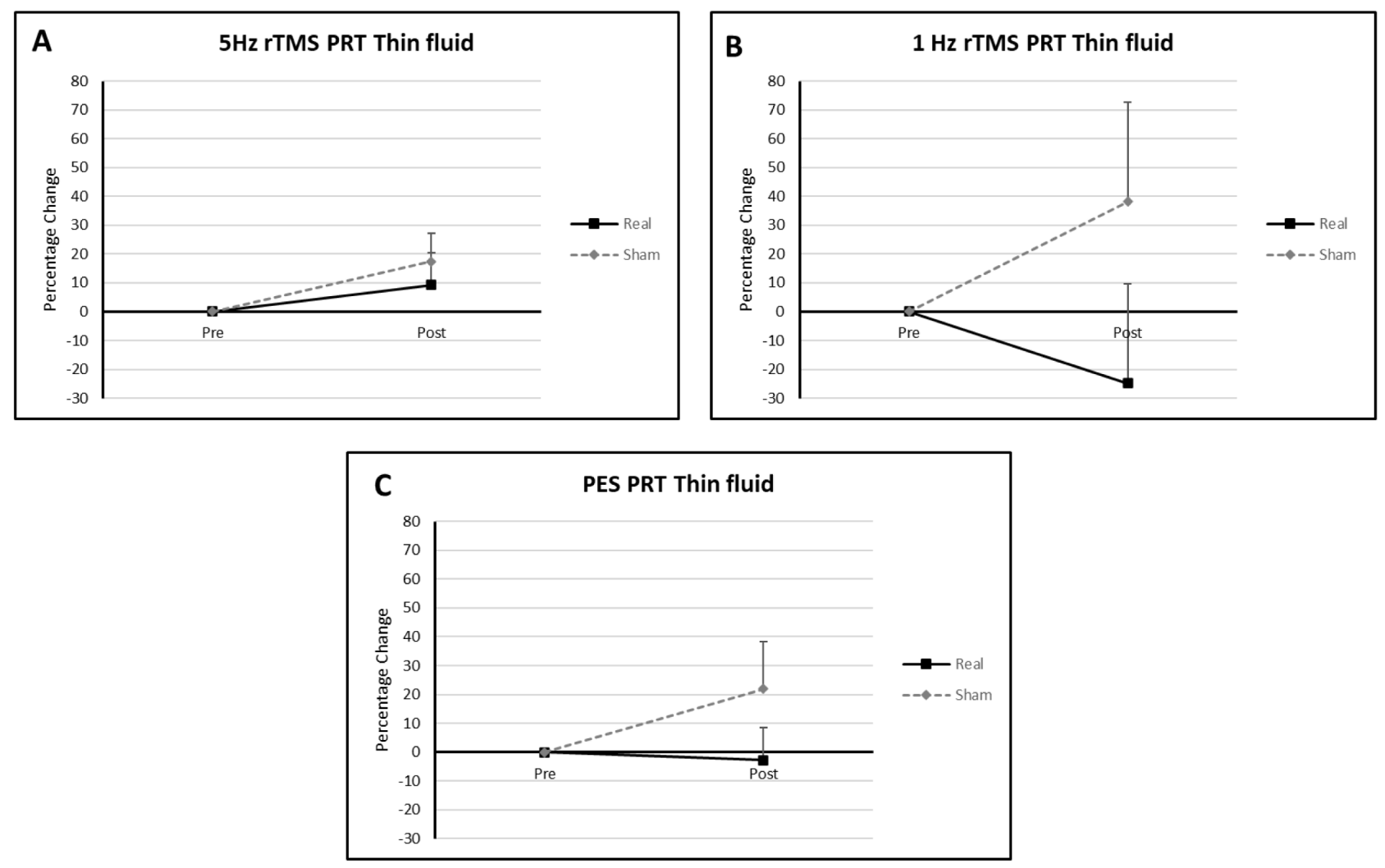

Figure 4. Graphs of percentage differences in PRT in the $1 \mathrm{~Hz}$ rTMS, $5 \mathrm{~Hz}$ rTMS and PES groups (A, B, C).

MEP

With regards PMEP amplitudes, despite the small number of participants in each interventional group, some separation of the trend lines began to emerge between sham and active stimulation for $1 \mathrm{~Hz}$ rTMS, $5 \mathrm{~Hz}$ rTMS and PES. In more detail, in the $5 \mathrm{~Hz}$ rTMS and PES interventional groups, interventions which have been shown to provoke cortical excitation within the swallowing motor system ${ }^{34,35}$, there was a trend towards greater PMEP amplitudes in the active treatment arms compared to sham. The opposite was the case following $1 \mathrm{~Hz}$ rTMS which is known to cause cortical suppression ${ }^{38}$. Were the groups larger, some significance may have eventually emerged. Despite no previous PD studies having been performed wherein rTMS was delivered to pharyngeal motor cortical swallowing areas (the 2019 Khedr study only stimulated the hand motor area ${ }^{25}$ ), these findings are tentatively supportive of the multiple studies which show high-frequency rTMS leads to increased PMEP amplitudes ${ }^{21,23,35}$. With regards to $1 \mathrm{~Hz}$ rTMS 

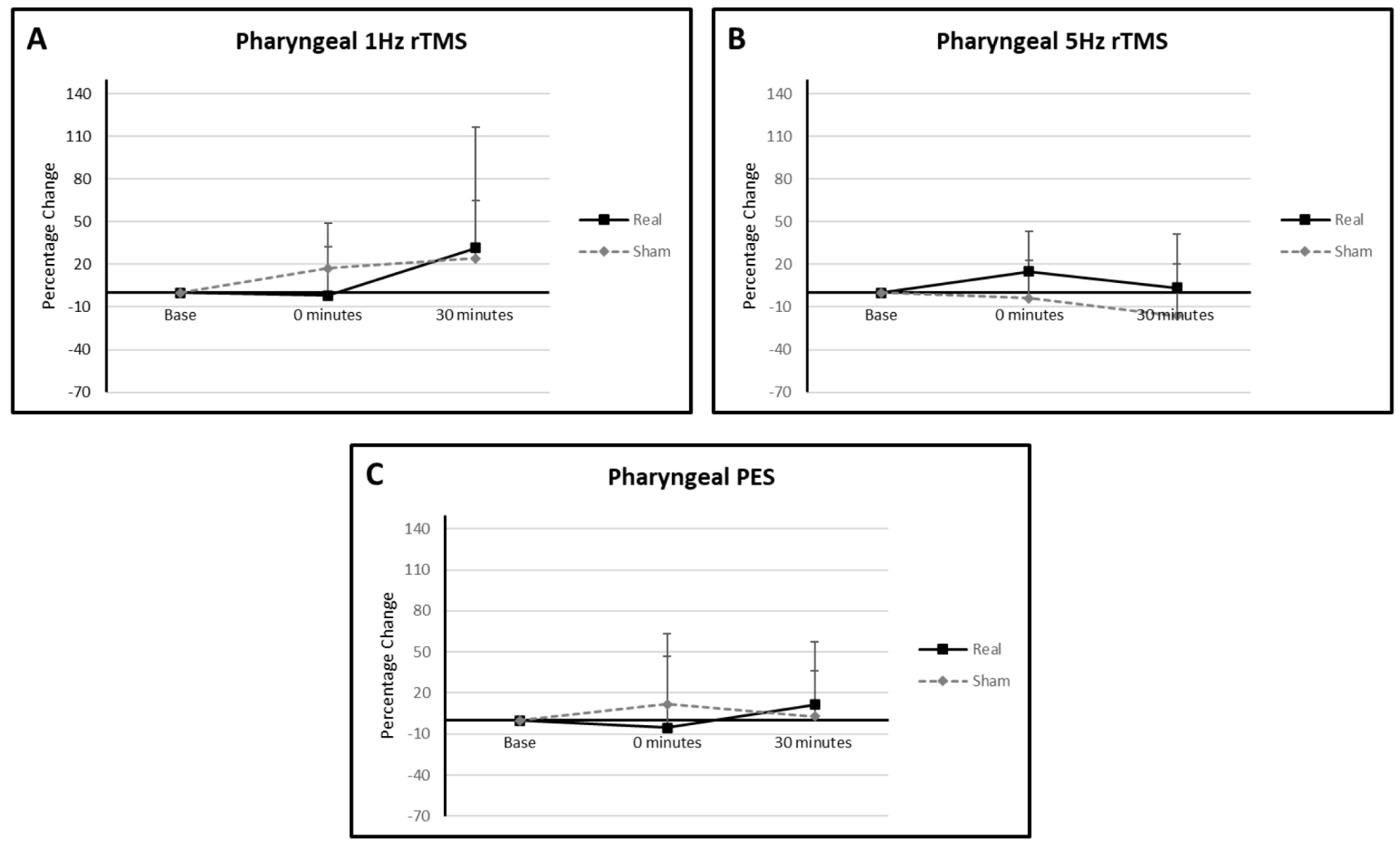

Figure 5. Graphs of percentage differences in PMEP amplitudes in the $1 \mathrm{~Hz}$ rTMS (A), (B) 5Hz rTMS and (C) PES interventional groups. Error bars illustrate standard deviations at each data point.

which has been shown to be suppressive when applied over the pharyngeal motor cortex ${ }^{36}$, there was some suggestion that the sham group had greater PMEP amplitudes than the active group. However small numbers make drawing any conclusions from this, premature.

\section{PD symptom and ADL scores}

We did note that the $H \& Y$ score was higher in the $1 \mathrm{~Hz}$ rTMS interventional group than the other intervention arms. Despite participants being allocated at random, this indicates participants in the $1 \mathrm{~Hz}$ rTMS group had slightly more severe PD symptoms than those in the other groups. By contrast, there were no differences in Schwab and England ADL scores across any of the intervention arms. The significance of the Hoehn and Yahr differences is unclear given similar ADL performances between groups which implies that participants in the $1 \mathrm{~Hz}$ rTMS group were still as fit and able as participants in the other interventional groups.

\section{Limitations}

Our study has several limitations. First, the number of patients that were able to be recruited was small. Patient recruitment was negatively impacted by several issues many of which were logistical and not in the control of the research team. Some examples include: there was some anecdotal evidence that emerged during the study which suggested patients with moderate PD were not as troubled by their relatively mild dysphagia as they were by their limb motor symptoms. This may explain why relatively few patients reached out to the research team regarding study participation. Conversely, patients with severe dysphagia were often too frail to be studied in a laboratory setting.

Another limitation was the onset of COVID-19 pandemic and research restrictions that were put in place to prevent the spread of the virus. Swallowing research, especially research involving pharyngeal intubation, is potentially aerosol generating meaning patient recruitment was stopped more than 6 months prior to the planned end date. This reduction in recruitment lead to reduced power and hence contributed to difficulty in drawing definitive conclusions from the study

Lastly, MEP recordings were only made up to 30 minutes poststimulation. This was done to reduce the time patients had to be present in the laboratory thereby making the experience more tolerable and reducing dropout. However, most healthy participant neurophysiological studies which measure MEP amplitudes record for up to an hour post stimulation ${ }^{35,39}$. Furthermore, in these studies, maximal separation between interventional groups tends to occur at times between 30 and 60 minutes $^{31,39}$. Therefore, in only making recordings up to 30 minutes post intervention, any delayed effects of neurostimulation might be missed. 


\section{Conclusion}

In conclusion, the use of neurostimulation in patients with PD dysphagia is well tolerated and might lead to some improvements in swallowing function, however suboptimal recruitment precludes more definitive conclusions. Larger studies will be needed to further answer the important question of does neuromodulation improve swallowing in PD associated dysphagia, in this understudied area of medicine.

\section{Data availability}

Figshare. Parkinsons study data AOS.xlsx. DOI: https://doi. org/10.48420/14958540.v $1^{40}$

This project contains the following data:

Data from a feasibility pilot study of the effects of neurostimulation on dysphagia recovery in Parkinson's Disease
Data are available under the terms of the Creative Commons Zero "No rights reserved" data waiver (CC BY 4.0 Public domain dedication).

Figshare. Study Protocol: Exploring Novel Neurostimulation Based Therapies for Swallowing Impairments in Parkinson's Disease. DOI: https://doi.org/10.48420/14995077.v1 $1^{41}$

Figshare. CONSORT checklist for study "A feasibility pilot randomised controlled study of the effects of neurostimulation on dysphagia recovery in Parkinson's Disease" DOI: https://doi.org/10.48420/15082662.v1 $1^{42}$

Figshare. CONSORT flowchart for the study "A feasibility pilot randomised controlled study of the effects of neurostimulation on dysphagia recovery in Parkinson's Disease". DOI: https://doi.org/10.48420/15082674.v2 ${ }^{43}$
1. Martino $\mathrm{R}$, Candundo $\mathrm{H}$, van Lieshout $\mathrm{P}$, et al: Onset and progression factors in Parkinson's disease: A systematic review. Neurotoxicology. 2017; 61: 132-41. PubMed Abstract | Publisher Full Text

2. Mu L, Sobotka S, Chen J, et al.: Alpha-synuclein pathology and axonal degeneration of the peripheral motor nerves innervating pharyngeal muscles in Parkinson disease. J Neuropathol Exp Neurol. 2013; 72(2): 119-29. PubMed Abstract | Publisher Full Text | Free Full Text

3. Spillantini MG, Schmidt ML, Lee VM, et al.: Alpha-synuclein in Lewy bodies. Nature. 1997; 388(6645): 839-40. PubMed Abstract | Publisher Full Text

4. Shahmoradian SH, Lewis AJ, Genoud C, et al.: Lewy pathology in Parkinson's disease consists of crowded organelles and lipid membranes. Nat Neuros $\mathrm{C}$. 2019; 22(7): 1099-109.

PubMed Abstract | Publisher Full Text

5. Tarakad A, Jankovic J: Diagnosis and Management of Parkinson's Disease. Semin Neurol. 2017; 37(2): 118-26.

PubMed Abstract | Publisher Full Text

6. Hoehn MM, Yahr MD: Parkinsonism: onset, progression and mortality. Neurology. 1967; 17(5): 427-42. PubMed Abstract | Publisher Full Text

7. de Rijk MC, Tzourio C, Breteler MM, et al:: Prevalence of parkinsonism and Parkinson's disease in Europe: the EUROPARKINSON Collaborative Study. European Community Concerted Action on the Epidemiology of Parkinson's disease. J Neurol Neurosurg Psychiatry. 1997; 62(1): 10-5. PubMed Abstract | Publisher Full Text | Free Full Text

8. de Rijk MC, Breteler MM, Graveland GA, et al.: Prevalence of Parkinson's disease in the elderly: the Rotterdam Study. Neurology. 1995; 45(12): 2143-6. PubMed Abstract | Publisher Full Text

9. Tjaden K: Speech and Swallowing in Parkinson's Disease. Top Geriatr Rehabil. 2008; 24(2): 115-26.

PubMed Abstract | Publisher Full Text | Free Full Text

10. Kalf JG, de Swart BJM, Bloem BR, et al.: Prevalence of oropharyngeal dysphagia in Parkinson's disease: a meta-analysis. Parkinsonism Relat Disord. 2012: 18(4): 311-5. PubMed Abstract | Publisher Full Text

11. Takizawa C, Gemmell E, Kenworthy J, et al.: A Systematic Review of the Prevalence of Oropharyngeal Dysphagia in Stroke, Parkinson's Disease, Alzheimer's Disease, Head Injury, and Pneumonia. Dysphagia. 2016; 31(3): 434-41.

PubMed Abstract | Publisher Full Text

12. Jenkins IH, Fernandez W, Playford ED, et al.: Impaired activation of the supplementary motor area in Parkinson's disease is reversed when akinesia is treated with apomorphine. Ann Neurol. 1992; 32(6): 749-57. PubMed Abstract | Publisher Full Text

13. Londos E, Hanxsson O, Alm Hirsch I, et al:: Dysphagia in Lewy body dementia - a clinical observational study of swallowing function by videofluoroscopic examination. BMC Neurol. 2013: 13:140.

PubMed Abstract | Publisher Full Text | Free Full Text

14. Easterling CS, Robbins E: Dementia and Dysphagia. Geriatr Nurs. 2008; 29(4): 275-85.

PubMed Abstract | Publisher Full Text

15. Cereda E, Cilia R, Klersy C, et al.: Swallowing disturbances in Parkinson's disease: a multivariate analysis of contributing factors. Parkinsonism Relat Disord. 2014; 20(12): 1382-7.

PubMed Abstract | Publisher Full Text

16. Karkos PD, Papouliakos S, Karkos CD, et al.: Current evaluation of the dysphagic patient. Hippokratia. 2009; 13(3): 141-6. PubMed Abstract | Free Full Text

17. Mansouri A, Taslimi S, Badhiwala JH, et al.: Deep brain stimulation for Parkinson's disease: meta-analysis of results of randomized trials at varying lengths of follow-up. J Neurosurg. 2018; 128(4): 1199-213. PubMed Abstract | Publisher Full Text

18. Restivo DA, Hamdy S: Pharyngeal electrical stimulation device for the treatment of neurogenic dysphagia: technology update. Med Devices (Auckl). 2018; 11: 21-6.

PubMed Abstract | Publisher Full Text | Free Full Text

19. Wassermann EM: Risk and safety of repetitive transcranial magnetic stimulation: report and suggested guidelines from the International Workshop on the Safety of Repetitive Transcranial Magnetic Stimulation, June 5-7, 1996. Electroencephalogr Clin Neurophysiol. 1998; 108(1): 1-16. PubMed Abstract | Publisher Full Text

20. Siebner HR, Rossmeier C, Mentschel C, et al:: Short-term motor improvement after sub-threshold 5 - $\mathrm{Hz}$ repetitive transcranial magnetic stimulation of the primary motor hand area in Parkinson's disease. J Neurol Sci. 2000 178(2): 91-4.

PubMed Abstract | Publisher Full Text

21. Verin $E$, Michou E, Leroi AM, et al.: "Virtual" lesioning of the human oropharyngeal motor cortex: a videofluoroscopic study. Arch Phys Med Rehabil. 2012; 93(11): 1987-90. PubMed Abstract | Publisher Full Text

22. Bath PM, Lee HS, Everton LF: Swallowing therapy for dysphagia in acute and subacute stroke. Cochrane Database Syst Rev. 2018; 10(10): CD000323. PubMed Abstract | Publisher Full Text | Free Full Text

23. Michou E, Mistry S, Jefferson S, et al:: Characterizing the mechanisms of central and peripheral forms of neurostimulation in chronic dysphagic stroke patients. Brain Stimul. 2014; 7(1): 66-73. PubMed Abstract | Publisher Full Text | Free Full Text

24. Scutt $P$, Lee HS, Hamdy S, et al.: Pharyngeal Electrical Stimulation for Treatment of Poststroke Dysphagia: Individual Patient Data Meta-Analysis of Randomised Controlled Trials. Stroke Res Treat. 2015; 2015: 429053. PubMed Abstract | Publisher Full Text | Free Full Text

25. Khedr EM, Mohamed KO, Soliman RK, et al.: The Effect of High-Frequency 
Repetitive Transcranial Magnetic Stimulation on Advancing Parkinson's Disease With Dysphagia: Double Blind Randomized Clinical Trial.

Neurorehabil Neural Repair. 2019; 33(6): 442-52.

PubMed Abstract | Publisher Full Text

26. Wagle Shukla A, Shuster JJ, Chung JW, et al.: Repetitive Transcranial Magnetic Stimulation (rTMS) Therapy in Parkinson Disease: A Meta-Analysis. PM $R$. 2016; 8(4): 356-66.

PubMed Abstract | Publisher Full Text | Free Full Text

27. Yang $C$, Guo Z, Peng $\mathrm{H}$, et al.: Repetitive transcranial magnetic stimulation therapy for motor recovery in Parkinson's disease: A Meta-analysis. Brain Behav. 2018; 8(11): e01132.

PubMed Abstract | Publisher Full Text | Free Full Text

28. Schwab JF, England AC: Projection technique for evaluating surgery in Parkinson's disease. Proceedings of the Third Symposium on Parkinson's Disease; Edinburgh: Churchill Livingstone, 1969.

29. Steele CM, Grace-Martin K: Reflections on Clinical and Statistical Use of the Penetration-Aspiration Scale. Dysphagia. 2017; 32(5): 601-16. PubMed Abstract | Publisher Full Text | Free Full Text

30. Bohning DE, Shastri A, McConnell KA, et al:: A combined TMS/fMRI study of intensity-dependent TMS over motor cortex. Biol Psychiatry. 1999; 45(4): $385-94$

PubMed Abstract | Publisher Full Text

31. Sasegbon A, Smith CJ, Bath PM, et al.: The effects of unilateral and bilateral cerebellar rTMS on human pharyngeal motor cortical activity and swallowing behavior. Exp Brain Res. 2020; 238(7-8): 1719-1733. PubMed Abstract | Publisher Full Text | Free Full Text

32. Sasegbon A, Niziolek N, Zhang M, et al:: The Effects of Midline Cerebellar rTMS on Human Pharyngeal Cortical Activity in the Intact Swallowing Motor System. Cerebellum. 2021; 20(1): 101-115. PubMed Abstract | Publisher Full Text | Free Full Text

33. Gow D, Rothwell J, Hobson A, et al.: Induction of long-term plasticity in human swallowing motor cortex following repetitive cortical stimulation. Clin Neurophysiol. 2004; 115(5): 1044-51. PubMed Abstract | Publisher Full Text

34. Jayasekeran V, Singh S, Tyrrell P, et al.: Adjunctive functional pharyngeal electrical stimulation reverses swallowing disability after brain lesions. Gastroenterology. 2010; 138(5): 1737-46. PubMed Abstract | Publisher Full Text
35. Jefferson S, Mistry S, Michou E, et al:: Reversal of a virtual lesion in human pharyngeal motor cortex by high frequency contralesional brain stimulation. Gastroenterology. 2009; 137(3): 841-9, 9.e1. PubMed Abstract | Publisher Full Text

36. Sasegbon A, Watanabe M, Simons A, et al.: Cerebellar repetitive transcranial magnetic stimulation restores pharyngeal brain activity and swallowing behaviour after disruption by a cortical virtual lesion. J Physiol. 2019; 597(9): 2533-2546.

PubMed Abstract | Publisher Full Text | Free Full Text

37. Chou YH, Hickey PT, Sundman M, et al.: Effects of repetitive transcranial magnetic stimulation on motor symptoms in Parkinson disease: a systematic review and meta-analysis. JAMA Neurol. 2015; 72(4): 432-40. PubMed Abstract | Publisher Full Text | Free Full Text

38. Mistry S, Verin E, Singh S, et al.: Unilateral suppression of pharyngeal motor cortex to repetitive transcranial magnetic stimulation reveals functional asymmetry in the hemispheric projections to human swallowing. J Physiol. 2007; 585(Pt 2): 525-38.

PubMed Abstract | Publisher Full Text | Free Full Text

39. Vasant $D H$, Michou $E$, Mistry $S$, et al.: High-frequency focal repetitive cerebellar stimulation induces prolonged increases in human pharyngeal motor cortex excitability. J Physiol. 2015; 593(22): 4963-77.

PubMed Abstract | Publisher Full Text | Free Full Text

40. Sasegbon A: Parkinsons study data AOS.xIsx. University of Manchester. Dataset. 2021. http://www.doi.org/10.48420/14958540.v1

41. Sasegbon A: Study Protocol: Exploring Novel Neurostimulation Based Therapies for Swallowing Impairments in Parkinson's Disease. University of Manchester. Preprint. 2021. http://www.doi.org/10.48420/14995077.v1

42. Sasegbon A: CONSORT checklist for study "A feasibility pilot randomised controlled study of the effects of neurostimulation on dysphagia recovery in Parkinson's Disease". University of Manchester. Online resource. 2021. http://www.doi.org/10.48420/15082662.v1

43. Sasegbon A: CONSORT flowchart for the study "A feasibility pilot randomised controlled study of the effects of neurostimulation on dysphagia recovery in Parkinson's Disease". University of Manchester. Online resource. 2021

http://www.doi.org/10.48420/15082674.v2 


\section{Open Peer Review}

\section{Current Peer Review Status: X ?}

\section{Version 1}

Reviewer Report 01 December 2021

https://doi.org/10.21956/amrcopenres.14082.r26847

(C) 2021 Jones $C$. This is an open access peer review report distributed under the terms of the Creative Commons Attribution License, which permits unrestricted use, distribution, and reproduction in any medium, provided the original work is properly cited.

\section{Corinne A. Jones}

${ }^{1}$ Department of Neurology, Dell Medical School, The University of Texas at Austin, Austin, TX, USA

2 Department of Speech, Language, and Hearing Sciences, Moody College of Communication, The University of Texas at Austin, Austin, TX, USA

This study investigated the feasibility of one session of $1 \mathrm{~Hz}$ rTMS, $5 \mathrm{~Hz}$ rTMS, and PES to impact swallowing physiology in individuals with PD and dysphagia. Given the low number of patients recruited, the authors did not assess statistical differences but instead looked at trends. Please find my comments below.

General

1. This is a small point, but there is no dysphagia recovery in $P D$, given the progressive neurodegeneration. Consider changing the title of the study.

2. Be consistent with use of abbreviations (e.g., PAS vs PAs or rTMS vs RTMS) and be sure that each abbreviation is defined (e.g., RMT is not defined)

Abstract

3. The phrase "there was visual separation in a positive direction" does not have much meaning and does not fully capture the results, particularly those where there were no changes for the active group but there was worsening of function for the sham group (e.g., Figure 2)

Introduction

4. A major missing piece is the rationale for using rTMS and PES for PD-associated dysphagia. The fact that there haven't been any studies yet is not a strong enough rationale. What about PD would cause rTMS or PES to be successful?

5. Please state your hypotheses

Methods

6. Please consider reorganizing the Methods section for easier reading (e.g., move the Protocol 
section to be sooner)

7. How was PD diagnosis confirmed?

8. How were symptoms of dysphagia identified?

9. What bolus was scored for the PAS in the screening VFS?

10. Define the PAS, OTT, PTT, and PRT for reproducibility and for those readers who may not be familiar

11. PAS is not a gold standard, and has several limitations. Please remove the "gold standard" phrasing and discuss weaknesses of the PAS in the Discussion

12. The Cumulative PAS is not a commonly-used metric, and is misleading in this study, as 10 thin liquid boluses were collected but only 3 paste and 3 solid swallows.

13. What was the solid that was swallowed?

14. Was the fluoroscopy pulsed or continuous?

15. Please perform reliability testing for all of the videofluoroscopic metrics

16. The authors mention a criterion for statistical significance, but do not mention any actual statistical test performed

17. Consider reporting raw change for the PAS values, as the PAS is an ordinal scale

18. Were the patients in the ON or OFF state of their PD medication?

19. The intervention is not clear in Figure 1. Please add more detail. Did each patient receive active and sham stimulation? If so, how long between sessions?

20. What was the criteria for 'visual improvement'?

Results

21. The percent change of PAS in Figure 2 does not match the mean or median change in Table 2.

Please explain.

22. Please present the pre and post MEPs in Table 4

Discussion

23. The statement about tolerance belongs in the Results. Also, what was assessed specifically in regards to tolerance?

24. How can the worsening of function in the sham group (figures 2-4) be described?

25. There does not appear to be much change in the MEP, despite what the authors report. 
26. Another large limitation that the authors do not address is the single session nature of the study. The previous neurostimulation papers that are referenced have a series of sessions of neurostimulation, which would be more ecologically valid

27. COVID-19 has negatively affected many swallowing projects, but it is not clear why recruitment was ended so early for this study

\section{References}

1. Steele CM, Grace-Martin K: Reflections on Clinical and Statistical Use of the PenetrationAspiration Scale.Dysphagia. 2017; 32 (5): 601-616 PubMed Abstract | Publisher Full Text

2. Borders JC, Brates D: Use of the Penetration-Aspiration Scale in Dysphagia Research: A Systematic Review.Dysphagia. 35 (4): 583-597 PubMed Abstract | Publisher Full Text

Is the work clearly and accurately presented and does it cite the current literature? Partly

Is the study design appropriate and is the work technically sound?

Partly

Are sufficient details of methods and analysis provided to allow replication by others? No

If applicable, is the statistical analysis and its interpretation appropriate? Partly

Are all the source data underlying the results available to ensure full reproducibility? Yes

Are the conclusions drawn adequately supported by the results? Partly

Competing Interests: No competing interests were disclosed.

Reviewer Expertise: Swallowing physiology; dysphagia rehabilitation; Parkinson's disease dysphagia; noninvasive neurostimulation

I confirm that I have read this submission and believe that I have an appropriate level of expertise to confirm that it is of an acceptable scientific standard, however I have significant reservations, as outlined above.

Author Response 30 Mar 2022

Ayodele Sasegbon, University of Manchester, Manchester, UK

Reviewer 2

General

1. This is a small point, but there is no dysphagia recovery in PD, given the progressive 
neurodegeneration. Consider changing the title of the study. The title has been changed to "A feasibility pilot study of the effects of neurostimulation on swallowing function in Parkinson's Disease".

2. Be consistent with the use of abbreviations (e.g., PAS vs PAs or rTMS vs RTMS) and be sure that each abbreviation is defined (e.g., RMT is not defined)

Thank you for your input. Changes have been made to ensure consistency. Resting motor threshold (RMT) was defined on page 5 of the initial paper.

Abstract

3. The phrase "there was visual separation in a positive direction" does not have much meaning and does not fully capture the results, particularly those where there were no changes for the active group but there was worsening of function for the sham group (e.g., Figure 2)

A change has been made to the text so it now reads "there was small but visible difference in the outcomes between active and sham".

Introduction

4. A major missing piece is the rationale for using rTMS and PES for PD-associated dysphagia. The fact that there haven't been any studies yet is not a strong enough rationale. What about PD would cause rTMS or PES to be successful?

PD is a cause of neurogenic dysphagia. A means by which PD leads to dysphagia is neuronal damage to cortical swallowing centres. This damage causes a measurable reduction in activity over cortical swallowing centres. While the disease and mechanism of damage are different in post-stroke dysphagia, its effects are also caused by damage to cortical swallowing centres. As rTMS and PES are two techniques which have been shown to be effective at improving swallowing function in poststroke dysphagia by modulating the activity of cortical swallowing centres thereby encouraging beneficial neuroplastic changes, it was hypothethised that similar beneficial (compensatory) swallowing changes could be induced in patients with PD associated dysphagia. Furthermore, as stated in the introduction, rTMS has been used, to moderate effect, to treat PD motor symptoms affecting the limbs. In this context, both inhibitory $1 \mathrm{~Hz}$ and excitatory $5 \mathrm{~Hz}$ rTMS have been shown to be effective. As a result, it was not unreasonable to hypothesise that dysphagia may also be amenable to being ameliorated using neuromodulatory techniques such as rTMS and PES. This has been further clarified in the text.

5. Please state your hypotheses

This has been added to the text

Methods

6. Please consider reorganizing the Methods section for easier reading (e.g., move the Protocol section to be sooner)

The methods section has been reorganised

7. How was PD diagnosis confirmed? 
Only patients with a documented diagnosis of PD from a Neurologist were recruited. Furthermore, the diagnosis had to have been made at least two years prior to the start of the study. This information was detailed on page 3 of the first version of the paper.

8. How were symptoms of dysphagia identified?

On page 3 of the first version of the paper, patients were initially recruited based on self-reported symptoms of dysphagia. These would later be confirmed or refuted based on their initial videofluroscopy.

9. What bolus was scored for the PAS in the screening VFS?

On page six of the first version of the paper we explained that the PAS with the highest numerical value was recorded for each bolus (in the case of multiple swallows) before being added together for each category (thin fluid, paste, solid and cup drinking). This has been stated in other parts of the text for additional clarity.

10. Define the PAS, OTT, PTT, and PRT for reproducibility and for those readers who may not be familiar

These have been defined in the text for clarity.

11. PAS is not a gold standard, and has several limitations. Please remove the "gold standard" phrasing and discuss weaknesses of the PAS in the Discussion

References to PAS as a gold standard have been removed from the text. However, it is true to say that PAS is one of the most validated tools for the assessment of dysphagia in the field.

12. The Cumulative PAS is not a commonly-used metric, and is misleading in this study, as 10 thin liquid boluses were collected but only 3 paste and 3 solid swallows.

cPAS is a valid metric (6) that has been used in similar studies of this type (7) and was not selected to mislead. As long as the number of swallows is stated ( 10 thin liquid, 3 paste and 3 solid), as has been done in the text, we believe there should be no undue confusion.

13. What was the solid that was swallowed?

On page 4 of the initial version of the paper, the solid that was swallowed was stated to be a biscuit covered in barium sulphate.

14. Was the fluoroscopy pulsed or continuous?

VFS was continuous. This has been added to the text for clarity

15. Please perform reliability testing for all of the videofluoroscopic metrics

VFS recordings were anonymised and independently analysed by IC, a qualified speech and language therapist who was blinded to the data being analysed. The metrics used - PAS, OTT, PTT, and PRT - have been used and validated in previous studies in neurogenic dysphagia. The performance of reliability testing was out of scope for this small feasibility study, and given that all VFS were analysed by one individual, there was no concern that there would be intra-rater variation. 
16. The authors mention a criterion for statistical significance, but do not mention any actual statistical test performed

Our original intention was to statistically analyse our data, but due to poor recruitment, it would have been inappropriate to subject the data we collected to statistical analysis due to the high risk oftype II errors.

References to statistical analysis have been removed from the text for clarity.

17. Consider reporting raw change for the PAS values, as the PAS is an ordinal scale The method of reporting percentage changes from individual baseline has been used in several papers published in the field $(8,9)$. We do not believe that the addition of another table or another layer of analyses looking at raw changes from individual baseline would provide any additional information with respect to the key study findings.

18. Were the patients in the ON or OFF state of their PD medication?

The patients were in the ON state at the time of being studied. This has been added to the text.

19. The intervention is not clear in Figure 1. Please add more detail. Did each patient receive active and sham stimulation? If so, how long between sessions?

Yes, patients received active and sham stimulation within their interventional groups. At least a week separated each session. This information was on page 6 of the initial version of the paper.

20. What was the criteria for 'visual improvement'?

The term visual improvement was meant to indicate there was the appearance of change in favour of the treatment arm, but this was not quantifiable statistically.

Results

21. The percent change of PAS in Figure 2 does not match the mean or median change in Table 2. Please explain.

The values in table 2 are raw cPAS values while the values used in figure 2 are percentage changes from baseline.

22. Please present the pre and post MEPs in Table 4

An expanded table has been added to the text.

Discussion

23. The statement about tolerance belongs in the Results. Also, what was assessed specifically in regards to tolerance?

Tolerance was assessed by asking participants after each session how they felt and if had any post-intervention after-effects. This has been moved to the results section.

24. How can the worsening of function in the sham group (figures 2-4) be described? A potential reason why sham stimulation resulted in higher PAS scores than active stimulation is due to patient fatigue during the course of a study session. This has 
been added to the discussion.

25. There does not appear to be much change in the MEP, despite what the authors report. Thank you for this point. The MEP changes observed were minor and this has been stressed in the text. However, despite the study's limitations, at $\mathbf{3 0}$ minutes post intervention, amplitudes in the active groups were consistently raised compared to that observed in the sham groups.

26. Another large limitation that the authors do not address is the single-session nature of the study. The previous neurostimulation papers that are referenced have a series of sessions of neurostimulation, which would be more ecologically valid

This limitation has been added to the text. However, this study was designed to detect immediate post neurostimulation neurophysiological and swallowing behavioural changes.

27. COVID-19 has negatively affected many swallowing projects, but it is not clear why recruitment was ended so early for this study

Swallowing related studies at the University of Manchester were suspended during the pandemic due to concerns about the podential generation of aerosols during experimentation. This coincided with the PhD student, who was helping conduct the study, completing his PhD and returning to full time clinical practice. As a result, recruitment was ended, as there was no further funding to support the study post the reopening of research at the University. The authors did not think it was necessary to add this amount of detail to the paper.

\section{REFERENCES}

1. Jahanshahi M, Jenkins IH, Brown RG, Marsden CD, Passingham RE, Brooks DJ. Selfinitiated versus externally triggered movements. I. An investigation using measurement of regional cerebral blood flow with PET and movement-related potentials in normal and Parkinson's disease subjects. Brain. 1995;118 ( Pt 4):913-33.

2. Valls-Solé J, Pascual-Leone A, Brasil-Neto JP, Cammarota A, McShane L, Hallett M. Abnormal facilitation of the response to transcranial magnetic stimulation in patients with Parkinson's disease. Neurology. 1994;44(4):735-41.

3. Jefferson S, Mistry S, Michou E, Singh S, Rothwell JC, Hamdy S. Reversal of a virtual lesion in human pharyngeal motor cortex by high frequency contralesional brain stimulation. Gastroenterology. 2009;137(3):841-9, 9.e1.

4. Wu AD, Fregni F, Simon DK, Deblieck C, Pascual-Leone A. Noninvasive brain stimulation for Parkinson's disease and dystonia. Neurotherapeutics. 2008;5(2):345-61.

5. Wagle Shukla A, Shuster JJ, Chung JW, Vaillancourt DE, Patten C, Ostrem J, et al. Repetitive Transcranial Magnetic Stimulation (rTMS) Therapy in Parkinson Disease: A MetaAnalysis. PM R. 2016;8(4):356-66.

6. Steele CM, Grace-Martin K. Reflections on Clinical and Statistical Use of the Penetration-Aspiration Scale. Dysphagia. 2017;32(5):601-16.

7. Vasant DH, Sasegbon A, Michou E, Smith C, Hamdy S. Rapid improvement in brain and swallowing behavior induced by cerebellar repetitive transcranial magnetic stimulation 
in poststroke dysphagia: A single patient case-controlled study. Neurogastroenterol Motil. 2019;31(7):e13609.

8. Sasegbon A, Watanabe M, Simons A, Michou E, Vasant DH, Magara J, et al. Cerebellar repetitive transcranial magnetic stimulation restores pharyngeal brain activity and swallowing behaviour after disruption by a cortical virtual lesion. J Physiol. 2019.

9. Vasant DH, Michou E, Mistry S, Rothwell JC, Hamdy S. High-frequency focal repetitive cerebellar stimulation induces prolonged increases in human pharyngeal motor cortex excitability. J Physiol. 2015;593(22):4963-77.

Competing Interests: As previously stated

Reviewer Report 08 September 2021

https://doi.org/10.21956/amrcopenres.14082.r26775

(C) 2021 Park J. This is an open access peer review report distributed under the terms of the Creative Commons Attribution License, which permits unrestricted use, distribution, and reproduction in any medium, provided the original work is properly cited.

\section{Jin Woo Park}

Department of Physical Medicine and Rehabilitation, Dongguk University Ilsan Hospital, Gyeonggido, South Korea

The design and protocol of this study are very well structured. This study aimed to investigate the effect of three modes of non-invasive neurostimulation on dysphagia in patients with Parkinson's disease and these types of studies have already been conducted on stroke patients. However, due to the lack of participants, it has a fatal drawback as a study that cannot be concluded using only simple descriptions without undergoing statistical verification. As this reviewer gave up research due to COVID-19, I fully understand the feelings of the researchers, but the value of a paper with a simple explanation is bound to decrease. An additional disappointment is that although it was based on previous studies that noninvasive neurostimulation had an effect on other motor functions, there seems to be a lack of detailed explanation of what mechanism can change the swallowing function. Parkinson's disease is distinctly different from stroke, and the explanation of its mechanism must also be different.

Is the work clearly and accurately presented and does it cite the current literature? Yes

Is the study design appropriate and is the work technically sound? Yes

Are sufficient details of methods and analysis provided to allow replication by others? Yes

If applicable, is the statistical analysis and its interpretation appropriate? 


\section{Not applicable}

Are all the source data underlying the results available to ensure full reproducibility? Yes

Are the conclusions drawn adequately supported by the results? Partly

Competing Interests: No competing interests were disclosed.

Reviewer Expertise: Neurorehabilitation especially dysphagia rehabilitation

I confirm that I have read this submission and believe that I have an appropriate level of expertise to state that I do not consider it to be of an acceptable scientific standard, for reasons outlined above.

Author Response 30 Mar 2022

Ayodele Sasegbon, University of Manchester, Manchester, UK

Reviewer 1

The design and protocol of this study are very well structured. This study aimed to investigate the effect of three modes of non-invasive neurostimulation on dysphagia in patients with Parkinson's disease and these types of studies have already been conducted on stroke patients. However, due to the lack of participants, it has a fatal drawback as a study that cannot be concluded using only simple descriptions without undergoing statistical verification. As this reviewer gave up research due to COVID-19, I fully understand the feelings of the researchers, but the value of a paper with a simple explanation is bound to decrease. An additional disappointment is that although it was based on previous studies that noninvasive neurostimulation had an effect on other motor functions, there seems to be a lack of detailed explanation of what mechanism can change the swallowing function. Parkinson's disease is distinctly different from stroke, and the explanation of its mechanism must also be different.

Thank you for your points. Unfortunately due to inadequate patient recruitment no definitive conclusions with regard to rTMS and PES effectiveness in Parkinsons Disease (PD) could be drawn. It would also be unreasonable to apply any formal statistics to these data, due to the small numbers of patients in each group, meaning that type II errors would be very likely. However, we still believe the study has benefit in demonsrating that these techniques can be applied safely in patients with PD. With regards to the mechanism through which non invasive neurostimulation can improve swallowing in patients with PD, the following has been added to the text: "In PD the nature of Lewy body deposition, neuronal damage and attempted neuroplastic compensation results in cortical areas with both decreased (1) and increased activity (2). Within the areas with decreased activity, damage can be said to have exceeded compensatory efforts while in areas with increased activity attempted compensation is ongoing but with unclear effectiveness. Cortical rTMS and PES have been hypothesised to encourage beneficial neuroplastic changes in the brains of 
patients with neurogenic dysphagia in two distinct ways. Firstly, high frequency (excitatory rTMS) or PES are excitatory and increase neuronal activity over cortical swallowing centres (3). This increase in activity can either reverse suppressed neuronal activity due to pathological damage, thereby restoring a more normal state of activity (4), or perhaps increase activity in non suppressed areas thereby acting as the trigger to encourage compensation for contralateral damage or restoration of normal activity in areas with disordered neuronal firing. Secondly, low frequency (suppressive rTMS) is thought to block maladaptive neuronal activity in the motor cortex thereby allowing beneficial neuroplastic changes to occur (5)." The latter may be helpful in PD where there may be excessive (disfunctional) excitation, leading to behavioural improvements.

Competing Interests: As previously stated 